\title{
Stem cell signature in glioblastoma: therapeutic development for a moving target
}

\author{
Ichiro Nakano, MD, PhD \\ Department of Neurological Surgery, James Comprehensive Cancer Center, The Ohio State University, Columbus, Ohio
}

Tumor heterogeneity of adult high-grade glioma (HGG) is recognized in 3 major subtypes based on core gene signatures. However, the molecular signatures and clinical implications of glioma stem cells (GSCs) in individual HGG subtypes remain poorly characterized. Recently genome-wide transcriptional analysis identified two mutually exclusive GSC subtypes with distinct dysregulated signaling and metabolic pathways. Analysis of genetic profiles and phenotypic assays distinguished proneural (PN) from mesenchymal (MES) GSCs and revealed a striking correlation with the corresponding PN or MES HGGs. Similar to HGGs with a MES signature, MES GSCs display more aggressive phenotypes both in vitro and in vivo. Furthermore, MES GSCs are markedly resistant to radiation as compared with PN GSCs, consistent with the relative radiation resistance of MES GBM compared with other subtypes. A systems biology approach has identified a set of transcription factors as the master regulators for the MES signature. Metabolic reprogramming in MES GSCs has also been noticed with the prominent activation of the glycolytic pathway, comprising aldehyde dehydrogenase (ALDH) family genes. This review summarizes recent progress in the characterization of the molecular signature in distinct HGG and GSC subtypes and plasticity between different GSC subtypes as well as between GSCs and non-GSCs in HGG tumors. Clinical implications of the translational GSC research are also discussed.

http://thejns.org/doi/abs/10.3171/2014.9.JNS132253

KEY WORDS cancer stem cells; GBM; brain tumor; tumor heterogeneity; clonal evolution; mesenchymal; oncology

A HALLMARK of high-grade gliomas (HGGs), including the most malignant grade, glioblastoma (GBM), is their resistance to current therapies, which leads to extremely poor prognosis. Even patients with well-demarcated tumors in noneloquent areas that allow maximal gross-total resection at surgery and respond well to irradiation and the first-round chemotherapies inevitably develop tumor regrowth/recurrence resulting in a dismal outcome. Therefore, a better understanding of the molecular mechanisms of such malignancy is warranted in order that we may eventually provide novel and effective therapies for patients.

Accumulated evidence has suggested that multiple genetic and metabolic pathways create intricate signaling networks to facilitate crosstalk between oncogenic and onco-metabolic pathways that contribute to cancer initiation and propagation and to therapy resistance. Recent genome-wide expression profiling and global DNA methylation analysis followed by clinical assessment have clearly separated HGGs into 3 distinct subtypes: proneural (PN), mesenchymal (MES), and classical (or proliferative) (Table 1). ${ }^{29,34,37}$ Among these 3 subtypes, approximately half of HGGs with the PN signature are associated with $\mathrm{CpG}$ island methylator phenotype $\left(\mathrm{CIMP}^{+}\right)$, mutations in the metabolic enzyme IDH1, and the onco-metabolite 2-hydroxyglutarate. ${ }^{24}$ Clinically, this group of HGGs, which tend to slowly progress from low-grade glioma to eventual GBM, was previously classified as secondary GBM. From the therapeutic standpoint, treatment plans for this subclass should be designed separately, given the significantly better prognosis compared with the rest of the HGG tumor subtypes. The remaining half of PN tumors are associated with PDGFFR $\alpha$ overexpression and/or amplification. ${ }^{7,34,37}$ Although these two PN subtypes show overlapping gene signatures, the therapeutic response and patient outcome appear to be distinct (for undetermined reasons). In contrast, MES tumors are non-CIMP, retain wild-type IDH1, and are associated with somatic mutations and/or deregulated expression in the NFI gene and its associated pathway. In contrast to this sharp difference in the altered

ABBREVIATIONS ALDH = aldehyde dehydrogenase; CIMP = CpG island methylator phenotype; CSC = cancer stem cell; EMT = epithelial-mesenchymal transition; GBM = glioblastoma; GSC = glioma stem cell; HGG = high-grade glioma; $M E S=$ mesenchymal; $N S C$ = neural stem cell; $P N=$ proneural.

SUBMITTED October 22, 2013. ACCEPTED September 29, 2014.

INCLUDE WHEN CITING Published online November 14, 2014; DOI: 10.3171/2014.9.JNS132253.

DISCLOSURE This work was supported by the following grants: American Cancer Society MRSG-08-108-01, NIH/NCI P01 CA163205, NIH/NINDS R01 NS083767, R21 CA175875, R21 CA135013, and R01 NS087913. 
TABLE 1. Adult HGG subtypes*

\begin{tabular}{|c|c|c|c|c|}
\hline Tumor Subtype & $\mathrm{CIMP}^{+} \mathrm{PN}$ & CIMP- PN & MES & Classical \\
\hline Frequency & $12 \%$ & $14 \%$ & $35 \%$ & $39 \%$ \\
\hline Age distribution & Young adult (mean 44.6 yrs) & $\begin{array}{l}\text { Young adult to adult (mean } 50.6 \\
\text { yrs) }\end{array}$ & $\begin{array}{l}\text { Adult (mean } \\
53.4 \mathrm{yrs} \text { ) }\end{array}$ & Old adult (mean 57.7 yrs) \\
\hline Prognosis & Relatively benign & Benign or poor? & Poor & Poor \\
\hline Sensitivity to therapy & Not resistant & Not resistant? & Resistant & Resistant \\
\hline $\begin{array}{l}\text { Gene signature \& methy- } \\
\text { lome }\end{array}$ & $\begin{array}{l}\text { Distinct from other } 3 \text { subtypes, } \\
\text { global gene methylation }\end{array}$ & Mutually exclusive pattern, lo & methylation & $\begin{array}{l}\text { Partially overlap w/ both PN \& } \\
\text { MES, low methylation }\end{array}$ \\
\hline Frequent gene mutation & IDH1, TP53, ATRX & $\begin{array}{l}\text { PDGFRA amplification, TP53, } \\
\text { CDKN2A deletion, CDK4 }\end{array}$ & NF1 & $\begin{array}{l}\text { EGFR (amplification or vlll mu- } \\
\text { tation), CDKN2A deletion }\end{array}$ \\
\hline $\begin{array}{l}\text { Altered signaling pathway } \\
\text { \& metabolome }\end{array}$ & IDH1-regulated metabolism & RTK & $\begin{array}{l}\text { STAT3, C/ } \\
\text { EBP } \beta, \text { TAZ }\end{array}$ & RTK-PI3K-AKT-mTOR \\
\hline
\end{tabular}

* Data for frequency and age distribution are based on Sturm et al..$^{34}$

gene expression patterns and signaling pathways between $\mathrm{PN}$ and MES tumors, the genetic and epigenetic changes that highlight classical HGGs are relatively modest, with the exception of the prominent signals in the EGFR pathway. ${ }^{34}$ Given that the gene signatures of PN and MES HGGs appear to be almost completely mutually exclusive, it is tempting to speculate that HGGs have a single axis of gene signature from PN to MES. Nonetheless, drawing a definitive conclusion requires more evidence. This open question aside, the subsequent therapeutic response of all these 3 subtypes is indistinguishably poor (except for $\mathrm{CIMP}^{+} \mathrm{PN}$ tumors). Despite this fact, given that the individual HGG subtypes appear to be dependent on distinct signaling pathways and onco-metabolic pathways, it is likely that therapeutic strategies for 3 distinct HGGs should be designed based on tumor subtype, instead of applying the same therapeutic regimen in all cases of HGG. The inclusion/exclusion criteria of the currently ongoing clinical trials, however, are mostly designed for GBM without separation into subtypes.

\section{Proneural and Mesenchymal GSC Subtypes}

Following the identification of these GBM subtypes, two papers ${ }^{5,26}$ published in 2013 identified two mutually exclusive distinct types of tumor cells with stem cell properties, termed glioma initiating/propagating cells or glioma stem cells (GSCs), from HGG tumors (Table 2 and Fig. 1). These two distinct GSC subtypes, proneural (PN) and mesenchymal (MES), were identified by means of genome-wide profiling and possess unique properties that are considered to contribute causally to the therapeutic resistance of the individual HGG subtypes. Given the distinct gene sets and signaling pathways that are differentially activated in each subtype of HGG, GSCs in each subtype may also harbor distinct and dysregulated pathways that are responsible for their unique phenotypes in tumor initiation, propagation, and subsequent resistance to therapies. Thus, it is essential to advance the molecular understanding of tumor-initiating GSCs in each subtype so that establishment of the tailored targeting strategies for individual HGGs can eventually be accomplished. In addition, a GSC subtype with classical signature has not been convincingly identified. It is yet to be determined whether the discrepancy between HGG subtypes and GSC subtypes is simply due to a technical limitation (e.g., the current in vitro culture favoring either of the identified two but not all three GSC subtypes), or a scientific reason (e.g., the classical HGGs being derived from the mixed population of PN and MES GSCs and their progeny cells). Future studies would clarify this open question.

\section{Phenotypic Differences of the GSC Subtypes}

Phenotypically, these two GSC subtypes also appear to be markedly distinct. ${ }^{5,26}$ MES GSCs are more aggressive, invasive, angiogenic, and resistant to radiation treatment than PN GSCs both in vitro and in vivo. In addition, MES GSCs are predominantly derived from primary GBMs that arise de novo, whereas PN GSCs reside in both Grade III gliomas, particularly those with an oligodendrocytoma component, and GBMs. ${ }^{26}$ However, these data require careful interpretation. Favorable growth in a given condition in vitro does not necessarily mean that one group of tumor cells is clinically more aggressive than the other. One major limitation of the current in vivo growth assay using animals is the attenuated immune response due to the fact of xenografting human cells into immunocompromised animal brains. As literature suggests, immune response to tumor cells plays a substantial role in cancer growth and/or inhibition. In fact, various immune-related genes were specifically upregulated in the MES type of GSCs, which may suggest that the immune-compromised condition has some effect on growth of MES GSCs in one way or the other. This hypothesis is supported by the fact that response to the clinical trials of immunotherapy is significantly different between MES GBM and the rest of the subtypes. ${ }^{30}$ To address the difference in response to treatment, several translational research laboratories have recently established mouse genetically engineered GBM models with diverse tumor subtypes. These models may help us to understand the pathophysiology of the individual GSC subtypes (or even missing GSC subtypes) in a microenvironment with an intact immune system.

Evidence is accumulating that a minor subpopulation of the CD133-expressing GSCs in GBM may be more resis- 
TABLE 2. Glioma stem cell subtypes*

\begin{tabular}{|c|c|c|}
\hline \multirow[b]{2}{*}{ Characteristic } & \multicolumn{2}{|c|}{ Subtype } \\
\hline & PN & MES \\
\hline Frequency & $60-65 \%$ & $35-40 \%$ \\
\hline Original tumor grade & Grade III, oligodendrocytoma through GBM & Mostly GBM, some Grade III astrocytoma \\
\hline Sensitivity to therapy & Relatively sensitive to radiation & Radioresistant \\
\hline Growth kinetics in vivo & Relatively slow, similar to MES & Fast \\
\hline Stem cell marker & CD133, CD15 & ALDH1A3 (Aldefluor) \\
\hline Potential cell of origin & Embryonic-neonatal neural stem cell & Adult neural stem cell-differentiated astrocyte \\
\hline Gene ontogeny & Neurogenesis & Mesenchymal stem cell, immune system \\
\hline Altered signaling pathway & Notch, PDGFRA, GLUT3-mediated glucose uptake & NF-KB, ALDH1A3, glycolysis, inflammasome \\
\hline $\begin{array}{l}\text { Clinical trial related to } \\
\text { each subtype }\end{array}$ & $\begin{array}{l}\text { Notch/gamma-secretase inhibitor (RO4929097), } \\
\text { PDGFR inhibitor (sorafenib), PI3K-mTOR inhibi- } \\
\text { tors, virus therapy }\end{array}$ & $\begin{array}{l}\text { NF- } \kappa B \text { inhibitor (dexanabinol), immune thera- } \\
\text { py, glycolysis inhibitor (DCA) }\end{array}$ \\
\hline
\end{tabular}

* Data based on Bhat et al. ${ }^{5}$ and Mao et al. ${ }^{26}$

tant to radiation treatment than non-GSCs within the same tumors because of more robust and efficient activation of the DNA damage checkpoint response. ${ }^{2,18}$ However, the two GSC subtypes are distinguished by differing degrees of radiosensitivity. MES GSCs exhibit significantly higher radioresistance than PN GSCs, with marked upregulation of genes associated with DNA repair. In fact, Phillips et al. ${ }^{29}$ based on analysis of a large set of clinical data from HGG cases, presented evidence that at least some PN tumors recur in a MES state. Consistently, YKL40 and CD44 expression correlates with radioresistance and worse overall survival, ${ }^{6,28}$ as an enrichment marker for the MES subclass of GSCs and HGG tumors. This observation is analogous to the therapeutic insult-induced phenotypic changes of cancer cells termed epithelial-mesenchymal transition (EMT) (e.g., in breast, ${ }^{17,21}$ colon, ${ }^{31}$ and pancreatic ${ }^{14,38}$ cancers). Whether a specific GSC subpopulation activating signaling and metabolic pathways in response to therapies is the predominant driver for a global MES transdifferentiation in clinical tumors remains to
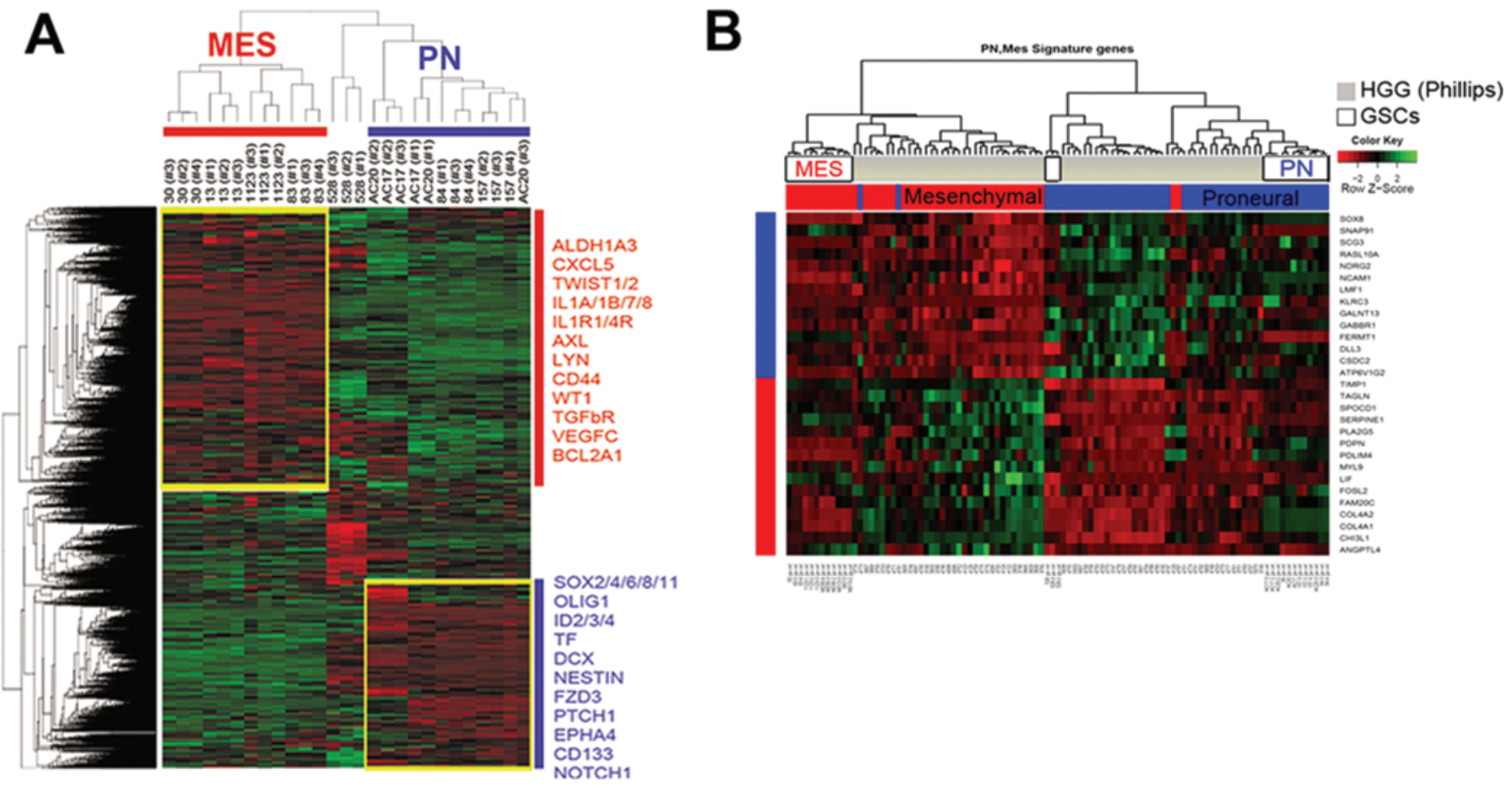

FIG. 1. A: Hierarchical biclustering of genes differentially expressed between PN and MES GSCs. B: Heat map with pairwise Pearson correlation for the Phillips HGG data set ${ }^{29}$ with the microarray samples. Stronger correlation is observed among microarray samples of the same type compared among and with Phillips' samples of the same subtype. These images were previously published in Mao P, Joshi K, Li J, Kim SH, Li P, Santana-Santos L, Luthra S, Chandran UR, Benos PV, Smith L, Wang M, Hu B, Cheng SY, Sobol RW, Nakano I: "Mesenchymal glioma stem cells are maintained by activated glycolytic metabolism involving aldehyde dehydrogenase 1A3." Proc Natl Acad Sci U S A. 110(21):8644-8649, 2013. 
be determined. Data in the study by Bhat et al..$^{5}$ showed that MES GSCs in vitro and MES GSC-derived mouse GBM-like tumors in vivo can repair DNA damage more efficiently, as evidenced by reduced $\gamma-\mathrm{H} 2 \mathrm{AX}$ foci in these cells, than can their counterparts with a PN trait. It could be argued that HGG cells derived from different cells of origin might vary in their capacity to overcome radiation insult (discussed more later).

\section{Clinical Significance}

Personalized medicine for cancer patients is yet to be realized, but molecular stratification-based prediction of therapeutic impact in GBM is evolving. One such example is the sensitivity of HGGs to the DNA alkylating agent temozolomide. The promoter methylation status of $O$ 6-methlylguanine-DNA methyltransferase (MGMT) indicates statistically significantly the sensitivity of treated tumors to temozolomide. ${ }^{15}$ Similarly, a set of genes has been shown to reliably predict the outcome of GBM patients and can potentially guide patients to investigate alternative, more exploratory therapeutic options for tumors that are refractory to current therapies..$^{11}$ Bhat et al. ${ }^{5}$ presented experimental data showing that the conventional antibiotic agent, minocycline, may represent one such candidate treatment for patients with GBM with the MES signature. This agent is clinically attractive because it is already approved by the FDA and has well-known pharmacokinetics and toxicity profiles, ${ }^{27}$ is off patent (which would minimize costs), and is known to cross the blood-brain barrier. ${ }^{39}$ In light of these observations, minocycline can possibly be a viable strategy for targeting at least the MES subclass of this devastating disease. Clearly, however, we should continue to explore more possibilities for therapeutic strategies for all the GBM subtypes.

\section{GSC Subtype-Specific Markers in Tumors}

Another hallmark of cancer stem cells (CSCs) is the ability to reinitiate and form cancers after completion of surgery and adjuvant therapies, which may directly indicate how long each tumor stays stable after therapy and the subsequent risk of recurrence of individual cases. Extensive studies in GSCs aimed at identifying such antigens have been undertaken primarily due to the mixed results using CD133, ,23,32,33 originally proposed to be the definitive marker for GSCs. ${ }^{32} \mathrm{CD} 15$ was also convincingly shown in numerous GSCs to be a tumor-initiating marker ${ }^{33}$ and other studies have shown a wide variety of markers (such as L1CAM, ${ }^{11}$ and integrin $\alpha 6^{20}$ ) that not only enrich for tumor cells harboring tumor-initiating potential, but also play a critical role in maintaining the GSC phenotype. Yet, none of these antigens has been proven to be a universal or exclusive marker for GSCs in HGG. In fact, PN HGGs appear to express CD133 and CD15 in their GSCs, whereas MES HGGs almost completely lack CD133 or CD15 expression, but instead, show increased expression of CD44, another cell surface marker that has also been shown to be enriched in CSCs in various other organs. ${ }^{6,22}$ Intriguingly, expression of any of these 3 molecules (CD133, CD15, or CD44) shows a statistically significant negative correlation with survival of patients with GBM. In other words, patients with tumors that express any of these markers at higher levels have poorer prognosis. Defining the correlation of each of these markers to GSCs or a subset of GSCs requires further study, but at least the genes that encode for them have value to predict clinical outcome.

Expression of a given lineage-specific antigen in distinct subsets of GSCs may have value beyond the role of a marker. For example, CD15 is expressed in both embryonic and adult neural stem cells (NSCs), which may indicate that the cell of origin of HGGs with a PN character is NSCs in either embryonic or adult brains. ${ }^{8}$ On the other hand, CD44 identifies an astrocyte-restricted precursor cell (ARP) that has lost the immature and multipotent state of the NSC and is committed to astrocyte lineages both in vitro and in vivo. ${ }^{25}$ In line with these observations, mature astrocytes have also been successfully transformed in mouse models of GBM. ${ }^{1}$ Collectively, mature astrocytes in the adult brain may represent the cell of origin for MES GBMs. In turn, a non-stem cell origin of oligodendrocytomas has been proposed..$^{22,23}$ In support of this notion, expression of additional cell surface markers such as PDGFR $\alpha$ and/or NG2, both specific for oligodendrocyte precursor cells (OPCs), has been reported in the oligodendrocytoma-derived GSCs. Collectively, the cells of origin for different subtypes of glioma are likely distinct.

\section{Distinct Oncogene Pathways Regulating Stem Cell Signatures in GBM Subtypes}

According to the study by Carro et al., a MES phenotype of glioma tumors is causal for glioma initiation and progression in association with an uncontrolled ability to invade and stimulate angiogenesis. ${ }^{9}$ Gene expression analysis identified that overexpression of the MES signature and loss of the PN signature co-segregate with poor outcome in GBM. Nonetheless, differentiation into the MES lineage is virtually undetectable in normal neural tissue throughout development. Thus, it is unclear whether phenotypic shift toward the MES lineage is an aberrant event that occurs during brain tumor progression or whether glioma cells recapitulate the rare MES plasticity of neural stem cells (NSCs). Aside from this open question, a recent systems biology approach effectively inferred the master regulators that choreograph MES transformation of glioma. ${ }^{6,911}$ For example, C/EBP $\beta$ and STAT3 are the two major master regulators necessary in HGG cells for MES transformation. MES transformation results from the concurrent activation of these two conflicting transcriptional regulators normally operating to drive opposing signals (neurogenesis by C/EBP $\beta$ vs gliogenesis by STAT3). This condition is intolerable for normal NSCs, whereas it operates to permanently drive the aberrant MES phenotype in the context of the genetic and epigenetic changes that accompany HGG initiation and establishment (e.g., EGFR amplification, AKT activation). Because expression of $\mathrm{C} /$ EBP $\beta$ and STAT3 in HGG is essential to maintain the tumor-initiating capacity and the ability to infiltrate into adjacent normal brain, the two tissue factors (TFs) provide important clues for diagnostic and pharmacological intervention. Consistent with this, the combined expression of 
$\mathrm{C} / \mathrm{EBP} \beta$ and STAT3 is linked to the MES trait of primary GBM and provides an excellent prognostic biomarker for tumor aggressiveness.

The studies describing two distinct GSC subtypes may indicate that the molecular signatures associated with GSCs are not static entities, but rather can be driven by paracrine factors such as TNF $\alpha$ that in turn trigger activation of master TFs, including NF- $\kappa$ B and STAT3, to drive the MES signature in a subset of GSCs. ${ }^{5}$ This observation is in agreement with studies pointing out that exogenous cytokines play prominent roles in tumor progression of HGG and other cancer types. The clinical significance of these findings is supported by the in vivo tumor inhibition by pharmacological elimination of these TFs. In particular, the NF- $\kappa \mathrm{B}$ family, including p65 and p50, represents the master regulators to activate numerous genes primarily involved in cell proliferation, inflammation, and anti-apoptosis of MES GSCs. A role for p65/p50 in tumor progression has also been widely studied in CSCs in various organs, owing primarily to the suspected crosstalk between inflammation and cancer., ${ }^{4} 13$ The transcription factor TAZ was identified as the third key mediator of MES GBMs. ${ }^{6}$ While aberrant activation of these master TFs is an attractive explanation to the global MES signature observed in GBM, how these factors act in a pleiotropic fashion remains to be clarified. Interestingly these TFs play prominent roles in the inflammatory response, and past studies have shown considerable crosstalk between these TFs. Consistently, the genome-wide gene signature showed higher expression of the inflammatome genes such as IL6, IL8 (CXCL8), ILIBIC, and CXCL2 in the MES subclass, further reinforcing the notion that interplay between the secretion of cytokines (perhaps due to increased necrosis more frequently observed in MES GBM) and inflammation-associated TFs drives the MES signature. ${ }^{5,26}$ However, it should be noted that MES GSCs show constitutively higher expression of these factors independent of microenvironment in a cell-autonomous fashion, and therefore additional mechanisms that sustain their expression in MES GSCs must exist.

\section{Metabolic Reprogramming in GSCs in GBM}

The pathway analysis with HGG-derived GSCs displays a striking difference in the gene sets associated with cellular metabolism between the two GSC subtypes. The genes involved in glycolysis and gluconeogenesis pathways, including ALDH family genes, in particular $A L$ $D H 1 A 3$, are significantly upregulated in MES GSCs compared with PN GSCs (Fig. 2). ${ }^{26}$ Activities of ALDH1A3, the enzyme that catalyzes the conversion of acetate from acetaldehyde, is markedly increased in MES GSCs but not PN GSCs. In addition, experimental inhibition of ALDH1A3 by a pharmacological inhibitor or short hairpin RNA (shRNA)-mediated gene silencing attenuates a gain of MES gene signature and its associated phenotype (e.g., in vitro growth and radioresistance). Together, these data suggest that subtypes of GSCs in clinical HGG tumor tissues are identifiable by their in vitro and in vivo behaviors as well as their global mRNA expression profiles. Upregulation of tumor metabolic pathways including ALDH1A3
Glycolysis/Glyconeogenesis pathway

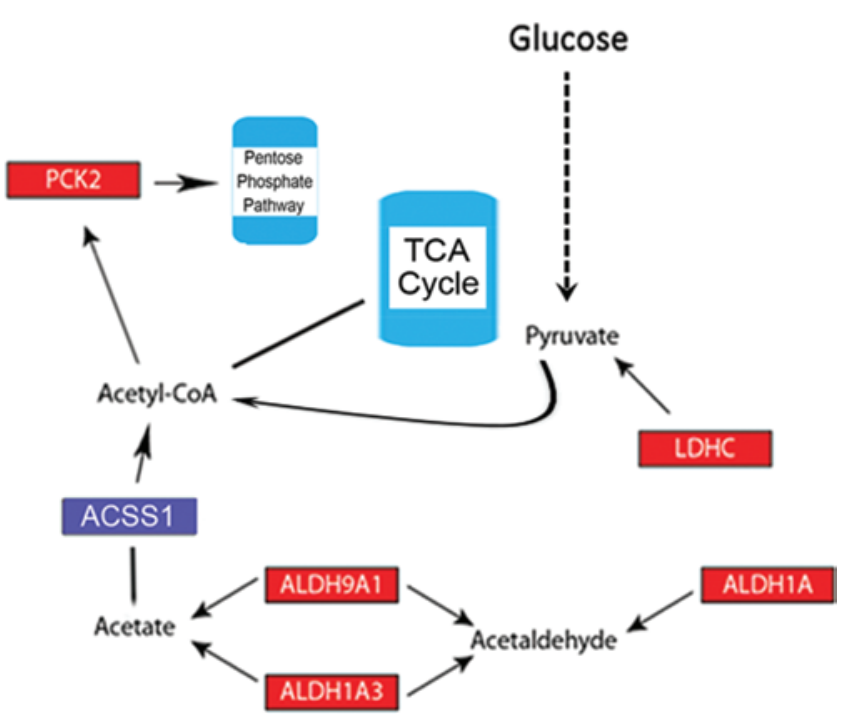

FIG. 2. The glycolysis/glyconeogenesis pathway containing aldehyde dehydrogenase genes (KEGG ID: hsa00010) was found to be significantly enriched in MES GSCs $(p=0.0001315) .{ }^{26}$ Genes in red were upregulated in MES compared with PN GSCs. KEGG = Kyoto Encyclopedia of Genes and Genomes; TCA = tricarboxylic acid. Image adapted from Mao P, Joshi K, Li J, Kim SH, Li P, Santana-Santos L, Luthra S, Chandran UR, Benos PV, Smith L, Wang M, Hu B, Cheng SY, Sobol RW, Nakano I: "Mesenchymal glioma stem cells are maintained by activated glycolytic metabolism involving aldehyde dehydrogenase 1A3." Proc Natl Acad Sci U S A. 110(21):8644-8649, 2013. Published with permission.

not only distinguishes MES GSCs from PN GSCs but also contributes to the persistent radioresistance of MES GSCs. Of note, ALDH1 has been recently recognized as a surrogate marker for CSCs in various organs and experimental ALDH1 elimination inhibited CSC growth and sensitized CSCs to chemotherapies in breast, ${ }^{12}$ colon, ${ }^{16}$ and lung ${ }^{35}$ cancers. Consistent with these reports, increased ALDH1A3 activity is associated with stem cell properties in MES GSCs. This observation is clinically relevant since immunohistochemistry of glial tumors shows that an increased proportion of ALDH1A3+ cells correlates with malignancy of glioma in the clinical setting and poor survival of patients. Furthermore, upregulation of ALDH1A3 could also be a potential biomarker to indirectly monitor lipid metabolism in gliomas. Activated cell metabolism (e.g., glycolysis) in cancer cells plays critical roles in rendering malignancy and poor responses to therapies of HGG ${ }^{32}$ In the clinic, ${ }^{18} \mathrm{~F}-\mathrm{FDG}$ PET is routinely used to evaluate increased glucose consumption and glycolysis in tumors. We found that ALDH1A9 and ALDH1A3, the enzymes that catalyze conversion of acetaldehyde into acetate, are markedly increased in MES GSCs. Detection of HGG by ${ }^{11} \mathrm{C}$-acetate PET based on increased activity of these two enzymes has recently been reported..$^{36}$ Thus, potential clinical application of ${ }^{11} \mathrm{C}$-acetate PET would also be useful for evaluation of subtypes of human HGGs as well as differential diagnosis of tumor recurrence versus radiation necrosis. 


\section{Plasticity of Two Subtypes of GSCs}

While the exact number of transcriptomic subtypes of GBM is still debatable, the PN and MES signatures represent robust mutually exclusive tumor subtypes, with other subtypes not as clearly demarcated. ${ }^{34}$ As described above, in some, if not all GBMs, tumor recurrence after failure of standard therapies is accompanied with a phenotypic shift from the PN to the MES subtype..$^{29} \mathrm{Lu}$ et al. ${ }^{25}$ showed EMTlike changes associated with use of the anti-angiogenic agent bevacizumab in preclinical mouse GBM models. Elevation of MES markers GBM specimens obtained in patients treated with bevacizumab was also demonstrated in this study. Regarding the molecular mechanism of action, activation of oncogenic receptor tyrosine kinase MET signals induced a program reminiscent of EMT in tumors and enhanced MES characteristics. Mao et al. ${ }^{26}$ described such an EMT-like phenotypic shift as also occurring in GSCs. Radiation treatment of PN GSCs downregulated PN markers and upregulated MES markers, suggesting radiationinduced EMT-like phenotypic changes in GSCs and their progeny. In contrast, radiosensitivity of individual GSCs did not show any statistically significant correlation with tumor grade, despite the distinct genetic background of WHO Grade III gliomas compared with Grade IV tumors (e.g., $1 \mathrm{p} / 19 \mathrm{q}$ loss of heterozygosity in malignant oligodendrogliomas). The observation of more PN GSCs being derived from Grade III tumors including oligodendrocytomas and more MES GSCs being derived from Grade IV GBMs further supports the significance of a MES signature, specifically in GSCs (Table 2). The molecular insight of this unique phenomenon warrants further investigation.

\section{Current Limitations and Future Direction}

Current studies have raised several open questions that will require further investigation. PN GSCs have been established from some recurrent GBM tumors after failure of whole brain radiation therapy. ${ }^{26}$ In turn, a small subset of CD44-expressing cells in PN GBM tumors display an MES signature, ${ }^{5}$ indicating that both PN and MES GSCs may coexist within the same tumors. These data may represent the intertumoral heterogeneity of GSCs, which then raise a possibility that therapies directed toward one subtype of GSCs can result in enrichment of the other, unrelated to the GSC plasticity.

The clinical significance of PN and MES signatures in HGGs also needs further in-depth investigation. Although contribution of the MES signature in glioma (and other cancers) to aggressiveness and poorer prognosis of affected patients is well recognized, a recent study by Sturm et al. ${ }^{34}$ reported that a small subset of PN GBMs, after removal of IDHI-mutant samples, has a worse prognosis. It is still debatable which subtype of gliomas has a better or worse prognosis and is responsive or nonresponsive to any given therapies.

One lesson from other CSC studies is that some stimulation (e.g., therapeutic insult) may induce a possible dedifferentiation ability of cancer cells that have otherwise lost the stem cell phenotype and have committed to differentiated non-tumorigenic cells. In fact, a recent study from the Pajonk laboratory ${ }^{19}$ demonstrated that ioniz-

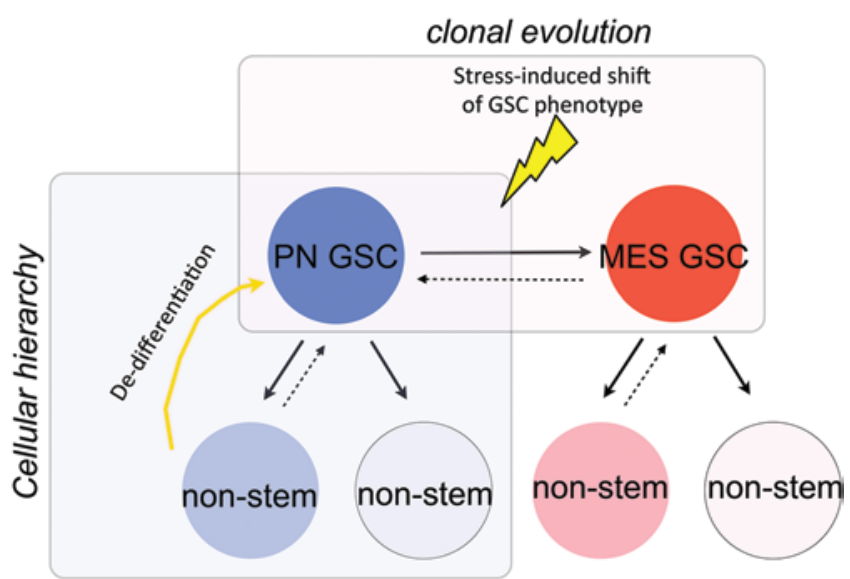

FIG. 3. Schema showing the combined theory of cellular hierarchy and clonal evolution to explain how glioma tumors establish heterogeneity. According to this theory, multiple clones of GSCs coexist in a single tumor. In a certain environment, non-stem cells are capable of acquiring stem cell signature, called "de-differentiation." In addition, cellular stress may induce transdifferentiation of one type of GSCs to another.

ing radiation treatment of breast cancer cells reactivates the expression of stem cell-related genes such as Oct4 (POU5F1) and SOX2 and induces a de novo CSC trait in otherwise non-tumorigenic breast cancer cells in a Notch signaling-dependent manner. Some GBM cells may have similar potential in a different molecular mechanism. If this is what is happening clinically, GBM cells that have lost stem cell properties may reactivate signals that induce a stem cell phenotype, resulting in reestablishment of the cellular hierarchy within the tumor (Fig. 3). A balanced therapeutic strategy targeting both GSCs and non-GSCs is then required for successful treatment of GBM. In addition to these strategies against GSCs and non-GSCs, a shift from one type of GSCs to another may have to be blocked simultaneously (Fig. 3). There is most likely no single definitive cellular target in HGG tumors to achieve cure of the disease. Rather, it is essential to eliminate both existing and de novo stem cell signatures, or a set of signatures, to provide hope for patients with HGGs.

\section{References}

1. Bachoo RM, Maher EA, Ligon KL, Sharpless NE, Chan SS, You MJ, et al: Epidermal growth factor receptor and Ink4a/ Arf: convergent mechanisms governing terminal differentiation and transformation along the neural stem cell to astrocyte axis. Cancer Cell 1:269-277, 2002

2. Bao S, Wu Q, McLendon RE, Hao Y, Shi Q, Hjelmeland $\mathrm{AB}$, et al: Glioma stem cells promote radioresistance by preferential activation of the DNA damage response. Nature 444:756-760, 2006

3. Beier D, Hau P, Proescholdt M, Lohmeier A, Wischhusen J, Oefner PJ, et al: CD133(+) and CD133(-) glioblastoma-derived cancer stem cells show differential growth characteristics and molecular profiles. Cancer Res 67:4010-4015, 2007

4. Ben-Neriah Y, Karin M: Inflammation meets cancer, with $\mathrm{NF}-\kappa \mathrm{B}$ as the matchmaker. Nat Immunol 12:715-723, 2011

5. Bhat KP, Balasubramaniyan V, Vaillant B, Ezhilarasan R, Hummelink K, Hollingsworth F, et al: Mesenchymal differentiation mediated by NF- $\kappa \mathrm{B}$ promotes radiation resistance in glioblastoma. Cancer Cell 24:331-346, 2013 
6. Bhat KP, Salazar KL, Balasubramaniyan V, Wani K, Heathcock L, Hollingsworth F, et al: The transcriptional coactivator TAZ regulates mesenchymal differentiation in malignant glioma. Genes Dev 25:2594-2609, 2011

7. Brennan CW, Verhaak RG, McKenna A, Campos B, Noushmehr H, Salama SR, et al: The somatic genomic landscape of glioblastoma. Cell 155:462-477, 2013

8. Capela A, Temple S: LeX/ssea-1 is expressed by adult mouse CNS stem cells, identifying them as nonependymal. Neuron 35:865-875, 2002

9. Carro MS, Lim WK, Alvarez MJ, Bollo RJ, Zhao X, Snyder EY, et al: The transcriptional network for mesenchymal transformation of brain tumours. Nature 463:318-325, 2010

10. Cheng L, Wu Q, Huang Z, Guryanova OA, Huang Q, Shou $\mathrm{W}$, et al: L1CAM regulates DNA damage checkpoint response of glioblastoma stem cells through NBS1. EMBO J 30:800-813, 2011

11. Colman H, Aldape K: Molecular predictors in glioblastoma: toward personalized therapy. Arch Neurol 65:877-883, 2008

12. Ginestier C, Hur MH, Charafe-Jauffret E, Monville F, Dutcher J, Brown M, et al: ALDH1 is a marker of normal and malignant human mammary stem cells and a predictor of poor clinical outcome. Cell Stem Cell 1:555-567, 2007

13. Grivennikov SI, Greten FR, Karin M: Immunity, inflammation, and cancer. Cell 140:883-899, 2010

14. Güngör C, Zander H, Effenberger KE, Vashist YK, Kalinina T, Izbicki JR, et al: Notch signaling activated by replication stress-induced expression of midkine drives epithelialmesenchymal transition and chemoresistance in pancreatic cancer. Cancer Res 71:5009-5019, 2011

15. Hegi ME, Diserens AC, Gorlia T, Hamou MF, de Tribolet N, Weller M, et al: MGMT gene silencing and benefit from temozolomide in glioblastoma. N Engl J Med 352:997-1003, 2005

16. Huang EH, Hynes MJ, Zhang T, Ginestier C, Dontu G, Appelman $\mathrm{H}$, et al: Aldehyde dehydrogenase 1 is a marker for normal and malignant human colonic stem cells (SC) and tracks SC overpopulation during colon tumorigenesis. Cancer Res 69:3382-3389, 2009

17. Huber MA, Azoitei N, Baumann B, Grünert S, Sommer $\mathrm{A}$, Pehamberger $\mathrm{H}$, et al: NF- $\kappa \mathrm{B}$ is essential for epithelialmesenchymal transition and metastasis in a model of breast cancer progression. J Clin Invest 114:569-581, 2004

18. Jamal M, Rath BH, Tsang PS, Camphausen K, Tofilon PJ: The brain microenvironment preferentially enhances the radioresistance of CD133(+) glioblastoma stem-like cells. Neoplasia 14:150-158, 2012

19. Lagadec C, Vlashi E, Della Donna L, Dekmezian C, Pajonk F: Radiation-induced reprogramming of breast cancer cells. Stem Cells 30:833-844, 2012

20. Lathia JD, Gallagher J, Heddleston JM, Wang J, Eyler CE, Macswords J, et al: Integrin alpha 6 regulates glioblastoma stem cells. Cell Stem Cell 6:421-432, 2010

21. Lester RD, Jo M, Montel V, Takimoto S, Gonias SL: uPAR induces epithelial-mesenchymal transition in hypoxic breast cancer cells. J Cell Biol 178:425-436, 2007

22. Liu Y, Han SS, Wu Y, Tuohy TM, Xue H, Cai J, et al: CD44 expression identifies astrocyte-restricted precursor cells. Dev Biol 276:31-46, 2004

23. Lottaz C, Beier D, Meyer K, Kumar P, Hermann A, Schwarz J, et al: Transcriptional profiles of CD133+ and CD133glioblastoma-derived cancer stem cell lines suggest different cells of origin. Cancer Res 70:2030-2040, 2010

24. Lu C, Ward PS, Kapoor GS, Rohle D, Turcan S, Abdel-Wahab O, et al: IDH mutation impairs histone demethylation and results in a block to cell differentiation. Nature 483:474-478, 2012

25. Lu KV, Chang JP, Parachoniak CA, Pandika MM, Aghi MK,
Meyronet D, et al: VEGF inhibits tumor cell invasion and mesenchymal transition through a MET/VEGFR2 complex. Cancer Cell 22:21-35, 2012

26. Mao P, Joshi K, Li J, Kim SH, Li P, Santana-Santos L, et al: Mesenchymal glioma stem cells are maintained by activated glycolytic metabolism involving aldehyde dehydrogenase 1A3. Proc Natl Acad Sci U S A 110:8644-8649, 2013

27. Naline E, Sanceaume M, Toty L, Bakdach H, Pays M, Advenier C: Penetration of minocycline into lung tissues. Br J Clin Pharmacol 32:402-404, 1991

28. Pelloski CE, Mahajan A, Maor M, Chang EL, Woo S, Gilbert M, et al: YKL-40 expression is associated with poorer response to radiation and shorter overall survival in glioblastoma. Clin Cancer Res 11:3326-3334, 2005

29. Phillips HS, Kharbanda S, Chen R, Forrest WF, Soriano RH, $\mathrm{Wu}$ TD, et al: Molecular subclasses of high-grade glioma predict prognosis, delineate a pattern of disease progression, and resemble stages in neurogenesis. Cancer Cell 9:157-173, 2006

30. Prins RM, Soto H, Konkankit V, Odesa SK, Eskin A, Yong WH, et al: Gene expression profile correlates with T-cell infiltration and relative survival in glioblastoma patients vaccinated with dendritic cell immunotherapy. Clin Cancer Res 17:1603-1615, 2011

31. Sakuma K, Aoki M, Kannagi R: Transcription factors c-Myc and CDX2 mediate E-selectin ligand expression in colon cancer cells undergoing EGF/bFGF-induced epithelial-mesenchymal transition. Proc Natl Acad Sci U S A 109:77767781,2012

32. Singh SK, Hawkins C, Clarke ID, Squire JA, Bayani J, Hide $\mathrm{T}$, et al: Identification of human brain tumour initiating cells. Nature 432:396-401, 2004

33. Son MJ, Woolard K, Nam DH, Lee J, Fine HA: SSEA-1 is an enrichment marker for tumor-initiating cells in human glioblastoma. Cell Stem Cell 4:440-452, 2009

34. Sturm D, Witt H, Hovestadt V, Khuong-Quang DA, Jones DT, Konermann C, et al: Hotspot mutations in H3F3A and IDH1 define distinct epigenetic and biological subgroups of glioblastoma. Cancer Cell 22:425-437, 2012

35. Sullivan JP, Spinola M, Dodge M, Raso MG, Behrens C, Gao $\mathrm{B}$, et al: Aldehyde dehydrogenase activity selects for lung adenocarcinoma stem cells dependent on notch signaling. Cancer Res 70:9937-9948, 2010

36. Tsuchida T, Takeuchi H, Okazawa H, Tsujikawa T, Fujibayashi Y: Grading of brain glioma with 1-11C-acetate PET: comparison with 18F-FDG PET. Nucl Med Biol 35:171-176, 2008

37. Verhaak RG, Hoadley KA, Purdom E, Wang V, Qi Y, Wilkerson MD, et al: Integrated genomic analysis identifies clinically relevant subtypes of glioblastoma characterized by abnormalities in PDGFRA, IDH1, EGFR, and NF1. Cancer Cell 17:98-110, 2010

38. Wang Z, Li Y, Kong D, Banerjee S, Ahmad A, Azmi AS, et al: Acquisition of epithelial-mesenchymal transition phenotype of gemcitabine-resistant pancreatic cancer cells is linked with activation of the notch signaling pathway. Cancer Res 69:2400-2407, 2009

39. Yenari MA, Xu L, Tang XN, Qiao Y, Giffard RG: Microglia potentiate damage to blood-brain barrier constituents: improvement by minocycline in vivo and in vitro. Stroke 37:1087-1093, 2006

\section{Correspondence}

Ichiro Nakano, Department of Neurological Surgery, James Comprehensive Cancer Center, 385 Wiseman Hall, OSUCCC, Columbus, OH 43210. email: ichiro.nakano@osumc.edu. 